\title{
PRECISION CCD PHOTOMETRY OF THE HORIZONTAL BRANCH
}

\author{
A. G. Davis Philip \\ Union College and ISO
}

The Strömgren four-color system is well suited to the measure and analysis of horizontalbranch stars. The increased accuracy of the CCD photometric system and the ability to measure fainter stars in very crowded regions make the combination of the CCD system and four-color photometry an excellent one to study horizontal-branch stars in globular clusters.

Horizontal-branch stars have been found to occupy different regions in the four-color diagrams. One group follows the ZAHB line in the $y,(b-y)$ diagram and these stars are assumed to be stars that are evolving to the blue from their initial positions on the ZAHB. Then there is a group of stars found approximately 0.2 mags above the first group. These stars are assumed to be those stars that have evolved past the turn-around point and are now heading redward, eventually to the asymptotic giant branch. In M 15 and M 92 stars have been found in the central regions which are on the $\mathrm{HB}$, but which are somewhat fainter than the ZAHB stars.

In Fig. 1. the y vs (b-y) diagram for $M 92$ is shown. These obeservations were made at Kitt Peak National Observatory using the T2ka chip and the No. 2 0.9-m telescope. Nine of

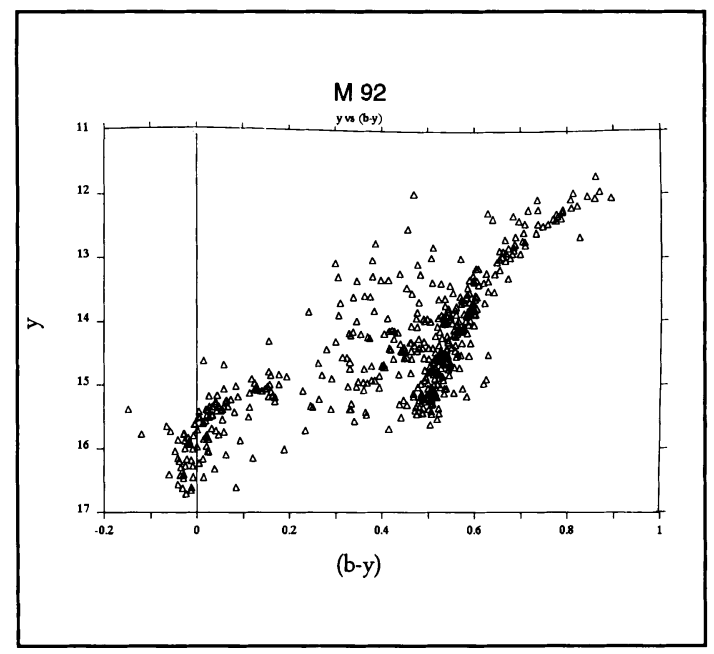

Fig. 1. Four-color CM diagram for M 92 (CCD Observations). 
the Sandage (1969) M 92 BHB stars had been set up as standards by observations made with a single channel photometer on the University of Arizona's 90-in telescope. Three frames were taken in each of the four filters. Dome flats were obtained for each filter. The data were reduced at the Dominion Astrophysical Observatory using Peter Stetson's programs, DAOPHOT and ALLSTAR (Stetson 1990). The internal rms errors of the $\mathrm{y}, \mathrm{b}, \mathrm{v}$ and $\mathrm{u}$ magnitudes was $\pm 0.016, \pm 0.014, \pm 0.017$ and \pm 0.018 for stars between $y=13$ and 17 mag.

In previous studies, using the smaller RCA chip, Philip (1990) showed that stars on the blue horizontal branch could be divided into two main groups. In a plot, combining observations of BHB stars in four different globular clusters, it was found that there was a lower envelope of stars, with a small scatter, running from right to left. Scattered above this relation ( $0.2 \mathrm{mag}$ or more) were a number of stars of higher luminosity. An analogy was made to a diagram from Sweigart (1987) in which $\log L$ was plotted vs $\log T_{\text {eff }}$ for various values of $\mathrm{Y}$ and $\mathrm{Z}$. Stars evolving to the blue all fell along the zero-age horizontal branch. Depending on the mass of the BHB star, the evolutionary track turned upward and to the right. The tightly grouped stars which form the lower envelope are assumed to be stars on the lower evolutionary track, evolving blueward. The more scattered group of stars above is assumed to be those stars which have evolved away from the ZAHB and are now tending toward the asymptotic red giant branch. In the M 92 observations (where the field of view included the entire cluster) it has been found that the stars fall in common groups in all the four-color diagrams, thus they form natural groups. ALLSTAR permits reductions of stars in the central region of the cluster and many new BHB stars have been found. There is also a group of stars that falls somewhat below the normal horizontal branch. Stars from both the inner and outer regions fall in this region.

A search has been made for an independent luminosity indicator that might confirm the photometric data. Gray (1991) has created an IBM PC based program, SPECTRUM, using the Kurucz (1991) models which allows one to enter a discrete set of temperatures, gravities and metallicities and the computer then plots the spectrum (from $\lambda 3900 \AA$ to $\lambda 4500 \AA$ ) at a spacing of $0.02 \AA$. A set of model grids was computed with SPECTRUM with parameters matching those of horizontal-branch stars and two possible luminosity indicators were found in the region of the $\mathrm{H} \gamma$ line (Philip 1994). In cooperation with Saul Adelman and Chris Aikman time for spectroscopic observations has been given at Kitt Peak National Observatory and the Dominion Astrophysical Observatory to test these luminosity indicators with observations of FHB stars on our observing lists.

\section{REFERENCES}

Gray, R. O. 1991 BAAS 23, 1382

Kurucz, R. L. 1991 in IAU Symposium No. 149, The Stellar Populations of Galaxies, A. Reis, ed., Kluwer Academic Pub, Dordrecht

Philip, A. G. D. 1990 in CCDs in Astronomy. II., A. G. D. Philip, D. S. Hayes and S. J. Adelman, eds., L. Davis Press, Schenectady, p. 107

Philip, A. G. D. 1994 in The MK Process at 50 Years, C. J. Corbally, R. O. Gray and R. F. Garrison, eds., ASP Conf. Series, 60, p. 148

Sandage, A. R. 1969 ApJ 157, 515

Stetson, P. B. 1990 PASP 99, 191 